Revue

Revue de l'histoire des religions

del'histoire des religions

2| 2007

Divination et révélation dans les mondes grec et romain

\title{
Vies et métamorphoses de la Sibylle
}

Notes critiques

Lives and Metamorphoses of the Sibyl

Jean-Michel Roessli

C OpenEdition

Journals

Édition électronique

URL : http://journals.openedition.org/rhr/5265

DOI : 10.4000/rhr.5265

ISSN : 2105-2573

Éditeur

Armand Colin

Édition imprimée

Date de publication : 1 avril 2007

Pagination : 253-271

ISBN : 978-2200-92333-4

ISSN : 0035-1423

\section{Référence électronique}

Jean-Michel Roessli, «Vies et métamorphoses de la Sibylle », Revue de I'histoire des religions [En ligne] 2 | 2007, mis en ligne le 01 juin 2010, consulté le 22 septembre 2020. URL : http:// journals.openedition.org/rhr/5265; DOI : https://doi.org/10.4000/rhr.5265

Tous droits réservés 


\section{Vies et métamorphoses de la Sibylle Notes critiques*}

Les études sur la Sibylle se sont multipliées au cours de ces dernières années, preuve, s'il en faut, de la vivacité de l'intérêt pour la divination et la poésie oraculaire. En raison de la permanence et de l'universalité de son message, la Sibylle, véritable réceptacle du divin, s'est prêtée à toutes sortes d'utilisations, exploitations et manipulations dans les domaines religieux et artistiques. Elle a inspiré poètes, peintres et compositeurs et alimenté les espérances de plusieurs groupes religieux. Les présentes notes critiques entendent profiter de parutions récentes pour rendre compte des réflexions actuelles sur la prophétesse antique et sa survie à travers les âges.

\section{Lives and Metamorphoses of the Sibyl}

Studies on the Sibyl have multiplied these last years, proof, if needed, of the liveliness of the question of divination and oracular poetry. Due to the long-lasting and broad influence of her message, the Sibyl, truthful recipient of divine communication, lent herself to many uses and appropriations in the areas of religion and the arts. She inspired poets, painters and composers and nourished the hopes of various religious groups. The present review article benefits from recent publications to report on current views about the ancient prophetess and her survival through the ages.

* À propos de : Monique Bouquet et Françoise Morzadec (éds.), La Sibylle. Parole et représentation, Rennes, Presses Universitaires de Rennes (PUR), 2004, 301 p., 19 figs. (Collection « Interférences »), 23 €. - Jackie Pigeaud, Les Sibylles. Actes des VIII Entretiens de La Garenne Lemot, 18 au 20 octobre 2001, Nantes 2005, 231 p., 5 pls. en couleur, $30 €$. 
Figure féminine de la divination et de la poésie, la Sibylle, au nom si mystérieux qu'il n'a pas encore livré tous ses secrets ${ }^{1}$, trouve son origine à l'époque archaïque, quelque part entre Orient et Asie Mineure. Depuis lors, elle n'a cessé de hanter l'imaginaire des Grecs, des Romains et de leurs héritiers en Occident, et cela depuis le haut Moyen Âge jusqu'à nos jours, en passant par la Renaissance et l'époque moderne.

Tour à tour une et multiple, païenne, juive et chrétienne, la Sibylle se fait porte-parole des polythéismes aussi bien que des monothéismes. Par les oracles qu'elle profère ou ceux qu'on lui prête, elle se fait encore l'écho des revendications identitaires de divers groupes humains, que ce soit les ambitions politiques d'une cité hellénistique ou l'affirmation de foi de quelque théologien juif

1. Les Anciens eux-mêmes, à l'instar de Varron (ap. Lactance, Institutions divines, I, 6, 7), Diodore de Sicile (Bibliothèque historique, IV, 66, 6) ou Servius (Commentaire à l'Énéide III, 445), ont tenté de lever le voile sur l'origine et la signification de ce nom. Leurs hypothèses, pour suggestives et séduisantes qu'elles sont, n'en demeurent pas moins indémontrables sur le plan philologique. Elles nourrissent l'imaginaire et font rêver les poètes, mais ne satisfont pas les linguistes. Les modernes, eux aussi, ont tenté de percer le mystère de ce nom, sans parvenir à des résultats plus probants (cf. Pierre Chantraine, Dictionnaire étymologique de la langue grecque. Histoire des mots, Paris, 1968, s. v. $\Sigma(\beta v \lambda \lambda \alpha$ ). Ils ont voulu y voir un mot d'origine tantôt grecque, tantôt sémitique et hésitent à y reconnaître au départ un nom propre appliqué par extension à une classe d'individus ou un nom commun qui se serait ensuite cristallisé sur un personnage particulier. Le fait que les sources les plus anciennes ne semblent connaître qu'une Sibylle incite toutefois à privilégier la première hypothèse. Sur tout cela, voir Herbert Chayyim Youtie, "Sambathis », Harvard Theological Review, 37 (1944), p. 209-218. Plus récemment, Valentin Nikiprowetzky, La Troisième Sibylle, Paris-La Haye, 1970, p. 11-16 ; Jean-Marc Rosenstiehl, Jean-Georges Heintz, « De Šibtu, la reine de Mari, à Sambéthé, la Sibylle chaldéenne ? ", Revue d'histoire et de philosophie religieuses, 52 (1972), p. 13-15. Pour d'autres hypothèses, voir encore Robert B. Coote, "Sibyl: 'Oracle", Journal of Northwest Semitic Languages, 5 (1977), p. 3-8 ; Jacob Hoftijzer, Karel Jongeling, Dictionary of the Northwest Semitic Inscriptions (Handbuch der Orientalistik. Erste Abteilung. Der Nahe und Mittlere Osten, 21), Leyde-New York, 1995, vol. 2, p. 774-775. 
ou chrétien. C'est sans doute pour répondre à tous ces besoins que la Sibylle s'est multipliée, de façon tantôt anarchique tantôt organisée, pour voir son nombre "canoniquement" fixé à "dix" dans le catalogue de Varron (antiquit. rer. div. frg. 56a Cardauns), puis à "douze" à la fin du Moyen $\hat{A}_{g e}{ }^{2}$. Ce sont les métamorphoses successives de cette prophétesse aux multiples visages, qui, de figure inspirée, est devenue inspiratrice pour les poètes, les artistes-peintres, les compositeurs et les érudits, que se propose d'aborder l'ouvrage publié par Monique Bouquet et Françoise Morzadec aux Presses Universitaires de Rennes. Ce livre réunit, en un ensemble varié mais cohérent, les vingt-et-une contributions, généralement brèves et synthétiques, d'un colloque organisé en octobre 2001 par le Centre d'Études des Littératures Antiques et Modernes de l'Université de Rennes 2-Haute-Bretagne.

Après un avant-propos rétrospectif sur les études rassemblées (p. 11-16), une première série de travaux est consacrée aux interprétations romaines de la Sibylle, telles qu'elles ressortent des œuvres de Virgile, Tibulle, Ovide, Silius Italicus et Servius, en passant par les libri Sibyllini et autres recueils d'oracles, dont la consultation était placée sous le strict contrôle de prêtres en charge du sacré (uiri sacris faciundis). Ces contributions sont opportunément précédées d'un article de Caroline Février sur Le double langage de la Sibylle : de l'oracle grec au rituel romain (p. 17-27), tant il est frappant de constater que la Sibylle a pu servir à la fois la divination inspirée des Grecs et la mantique inductive des Romains, s'exprimant pour cela aussi bien dans un langage débridé que dans un discours construit

2. Voir notamment mon «Catalogues de sibylles, recueil(s) de Libri Sibyllini et corpus des Oracula Sibyllina. Remarques sur la formation et la constitution de quelques collections oraculaires dans les mondes gréco-romain, juif et chrétien », dans Enrico Norelli (éd.), Recueils normatifs et canons dans l'Antiquité. Perspectives nouvelles sur la formation des canons juif et chrétien dans leur contexte culturel. Actes du colloque organisé dans le cadre du programme plurifacultaire La Bible à la croisée des savoirs de l'Université de Genève, 11-12 avril 2002 (Publications de l'Institut romand des sciences bibliques 3), Lausanne, 2004, p. 47-68 (avec une bibliographie abondante sur le sujet). 
et rétrospectivement adapté aux besoins de la politique religieuse de la République et de l'Empire. Mais l'auteur montre que ce double langage de la Sibylle se retrouve dans les libri Sibyllini eux-mêmes, dont le genre se situe à mi-chemin entre oracula et remedia, oscillant de l'un à l'autre en fonction des nécessités. On pourrait ajouter - mais c'est une évidence - que le langage de la Sibylle ne peut être que double, dans la mesure où celui-ci est souvent ambigu, équivoque, obscur, sibyllin pour tout dire. C. Février s'interroge enfin sur le moment où l'on a commencé à faire un usage politique de ces libri, sous les Tarquins à l'époque archaïque ou plus tardivement sous l'empereur Auguste. L'étude de Charles Guittard (Reflets étrusques sur la Sibylle, "Libri Sibyllini" et "Libri Vegoici", p. 29-42) revient ensuite sur la question longtemps débattue des racines étrusques de la prophétesse, en examinant le lien, suggéré par le commentaire de Servius ad Aen. VI, 72, entre la nymphe Vécu, Végoia ou Bégoé et la prophétie sibylline. Une belle traduction de l'oracle de Végoia est donnée en appendice de cet article, où un rapprochement est encore proposé avec l'oracle d'Hystaspe, à propos duquel je crois utile d'indiquer la contribution de Piero Franco Beatrice, «Le livre d'Hystaspe aux mains des chrétiens », dans Corinne Bonnet et André Motte (éds.), Les syncrétismes religieux dans le monde méditerranéen antique. Actes du colloque international en l'honneur de Franz Cumont à l'occasion du cinquantième anniversaire de sa mort, Bruxelles-Rome, 1999, p. 357-382, en complément de celle, fondamentale, de Bidez et Cumont, mentionnée par l'auteur.

Ensuite, ce sont les relectures ou appropriations plus spécifiquement littéraires de la Sibylle qui sont explorées. Jacqueline Champeaux se demande par exemple, dans son étude sur les Figures romaines de la Sibylle (p. 43-52), si le nom de la prophétesse n'est pas devenu une appellation générique pour désigner une figure étrange ou une vierge fanatique. Selon l'auteur, c'est à Virgile que l'on doit d'avoir « fondu en un seul personnage, à la forte identité, les figures multiples, donc fragmentaires, que lui avait léguées la tradition » (p. 47). Christophe Cusset s'intéresse à l'Alexandra de Lycophron (p. 53-60), dans laquelle il décèle une assimilation de Cassandre à la Sibylle, toutes deux vierges et toutes deux associées à une demeure souterraine 
décrite comme odieuse ${ }^{3}$. Dans son article intitulé La Sibylle et Médée : Virgile et la tradition argonautique (p. 61-68), Damien P. Nelis, s'inspirant du commentaire d'Eduard Norden et d'une réflexion de Hugh Lloyd-Jones, rapproche la Sibylle virgilienne et Médée, chacune d'elles étant prêtresse d'Hécate et rattachée de ce fait à des traditions catabatiques remontant jusqu'à l'Iliade (où Circé joue le rôle tenu ailleurs par Médée) et dont on trouve des traces dans les Argonautiques d'Apollonios de Rhodes. Albert Foulon traite ensuite des Sibylles élégiaques (p. 69-74), autrement dit des allusions littéraires à la Sibylle dans les œuvres d'Ovide, Properce, et surtout de Tibulle, dont il souligne l'originalité « dans l'importance accordée à la fois au personnage de la Sibylle et au contenu de son message, dépassant le seul destin d'Énée pour anticiper la domination de Rome sur tout l'univers » (p. 72). Alain Deremetz se penche de son côté sur La Sibylle dans la tradition épique à Rome : Virgile, Ovide et Silius Italicus (p. 75-83). L'auteur relève que la figure prophétique tutélaire de l'Énéide devient une « simple voix sans corps » dans les Métamorphoses et une friponne délurée dans les Punica. S'appuyant sur les théories du discours littéraire, il conclut que dans ces trois poèmes la Sibylle joue « un double rôle, celui de prophétesse des destins du héros au niveau de l'action racontée et celui d'un modèle poétique qui illustre le rapport de l'auteur à son œuvre » (p. 82).

Françoise Morzadec, l'une des deux éditrices du volume, consacre ensuite une étude pleine de finesse à Stace et la Sibylle : rivalité littéraire autour de la louange de Domitien, la Silve IV, 3 (p. 85-98). L'auteur s'efforce de comprendre et de définir les raisons qui ont poussé le poète à insérer un panégyrique à l'empereur dans le discours descriptif et visionnaire de la Sibylle. Pour Stace, à la différence de Virgile dont il s'inspire et se démarque tout à la fois,

3. De ce texte capital et fort énigmatique, nous disposons désormais de deux nouvelles traductions françaises assorties de notes et commentaires, l'une due à Gérard Lambin, éditée dans la même collection "Interférences » des Presses universitaires de Rennes en 2005, l'autre de Pascale Hummel, parue dans la Bibliothèque volante des éditions Comp'Act à Chambéry en 2006. Le même Christophe Cusset a organisé, en partenariat avec Evelyne Prioux, un colloque sur Lycophron qui s'est tenu à Lyon et Saint-Étienne du 18 au 20 janvier 2007. Inutile de dire que les Actes seront attendus avec impatience. 
la Sibylle est une figure littéraire plus qu'une prophétesse, et le but du poète est d'unir des éléments réalistes et imaginaires dans l'espace privilégié de la louange (p. 93). Monique Bouquet, coéditrice du volume, s'interroge pour sa part sur La Sibylle servienne, guide de l'exégèse moderne? (p. 109-118). Selon l'auteur, le célèbre commentaire de Servius "dépoétise" - en même temps qu'il "dépersonnalise", pourrait-on ajouter - la Sibylle, qui devient un nom "commun" et peut du même coup désigner «toute jeune fille dont le cœur reçoit la divinité » ( omnis puella cujus pectus numen recipit», In Aen. III, 445, p. 110). Pour M. Bouquet, «Servius serait à Virgile ce que la Sibylle est à Apollon » (p. 117). Autrement dit, le scholiaste de Virgile fonctionnerait à l'égard de l'œuvre qu'il commente un peu comme la Sibylle face au message du dieu Apollon dont elle se fait l'interprète. D'autre part, dans son commentaire, Servius semble suivre la distinction cicéronienne entre mantique «naturelle » et mantique « artificielle» (De divinatione, I, 18) et il décrit au moins trois phases dans l'histoire de la divination (Aen. VI, 46). D'Érythrées, la Sibylle se rend à Cumes dans le but d'obtenir d'Apollon une longue vie, qu'elle reçoit, mais sans corps, de sorte qu'elle finit par n'être plus qu'une voix (cf. Ovide, Métamorphoses, 14, 152-153). C'est en lien avec cette étude, mais pas seulement, qu'on lira l'article hautement instructif de Giuseppe Ramires sur Les additions italiennes dans l'épisode de la Sibylle de Cumes: Servius Ad Aen. VI, 37-135 (p. 119-129). L'auteur attire l'attention sur les supplementa Italica figurant dans certains manuscrits humanistes de Servius (le Parisinus Latinus 7965, écrit à Ferrare en 1469, par exemple), qui contiennent des interpolations parfois antérieures à eux, interpolations dues, selon V. Zabughin, « à la main de maître d'un helléniste éprouvé », mais non identifié (p. 120). Ramires fait une analyse herméneutique de ces interpolations, notamment celles de Pétrarque dans le codex Ambrosianus. Il examine ensuite quelques-unes des gloses relatives à la Sibylle dont on ne connaît aucun parallèle ailleurs et conclut qu'une étude globale de la question rendrait indispensable leur insertion dans le cadre d'une nouvelle édition critique du commentaire de Servius, édition qu'il juge du reste indispensable. Cet article, signé d'un 
auteur italophone, aurait encore gagné en lisibilité, s'il avait bénéficié d'ultimes retouches de la part des éditrices. Un peu plus tôt dans l'ouvrage, Sabina Crippa a livré d'intéressantes réflexions sur

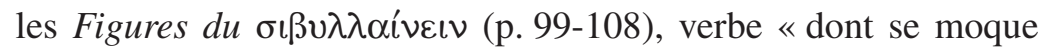
Aristophane et que Diodore de Sicile définit comme l'expression linguistique » désignant « le fait d'être inspiré » des dieux (p. 100). L'auteur attire en outre l'attention, comme elle avait déjà fait ailleurs, sur la distinction entre la Sibylle et les sibylles, de même qu'entre oralité et écriture du langage sibyllin, autrement dit entre vaticinations orales et prophéties mises par écrit. Dans cette contribution aussi, quelques italianismes, qu'il n'aurait pas été difficile de supprimer, subsistent dans le nom de quelques auteurs anciens (Erma, pour Hermas ; Ps.-Giustino, pour le Pseudo-Justin, etc.).

Ileana Chirassi Colombo, co-organisatrice et co-éditrice d'un autre colloque sur les sibylles ${ }^{4}$, s'arrête ensuite sur La bru de Noé (p. 131-149), titre inspiré d'un vers du troisième livre des Oracles sibyllins (v. 827 : «J'étais nymphe et de même sang que lui [Noé] », souvent traduit «J'étais sa bru et sa parente [de Noé]»), qui lie la Sibylle, figure préhistorique d'avant la chute de la Tour de Babel, à la famille de Noé. Cette Sibylle, juive sur les plans étymologique, lexicographique et métaphorique, est à proprement parler « la parole de Dieu ». Alternativement fille d'Ève ou de Circé, elle en vient à personnifier le Sabbat à l'époque hellénistique (p. 140). Sous le titre Quand Apollon s'est tu, les Sibylles parlent encore (p. 151-163), Nicole Belayche s'interroge sur le rôle tenu par les sibylles et leurs oracles dans les milieux païens et chrétiens des $\mathrm{III}^{\mathrm{e}}$ et IV $\mathrm{V}^{\mathrm{e}}$ siècles. Elle relève notamment que la transformation de la Sibylle, véritable "voyante" païenne, en une prophétesse quasichrétienne, se reflète dans le passage d'oracles proférés en état de transe à des prophéties de plus en plus sophistiquées et construites. L'auteur place son excellente étude sous le patronage du Discours de Constantin à l'assemblée des saints, dont elle ne remet pas en cause l'authenticité, pas plus qu'elle n'exprime de doute sur sa date

4. Ileana Chirassi Colombo, Tullio Seppilli (éds.), Sibille e linguaggi oracolari. Mito, storia, tradizione, Atti del Convegno Macerata-Norcia, Settembre 1994, Pise-Rome, 1998 (impr. 1999). 
de composition, le lieu où il a été prononcé et la circonstance pour laquelle il a été rédigé : à Antioche, le vendredi saint de l'année 325. N. Belayche n'ignore pourtant pas que la paternité de ce Discours - à laquelle je souscris également - ne fait pas l'unanimité, aujourd'hui encore (cf. Cataudella, 2001), parmi les spécialistes, mais il faut ajouter que la date de sa composition, comme le lieu et la circonstance dans lesquels il a été délivré font également l'objet de débats entre historiens, qui hésitent entre les vendredis saints de 313 et 325 et d'autres circonstances situées entre ces deux dates ${ }^{5}$. Quant au lieu où le Discours aurait été prononcé - s'il l'a jamais été, tout au moins sous la forme où nous le connaissons -, Antioche n'est pas la seule candidate en lice. On a également pensé à Nicomédie (Bleckmann, Barnes) et à Rome (Edwards). Or, ce point n'est pas sans importance dans l'argumentation de l'auteur, puisqu'elle associe étroitement le lieu du discours de l'empereur à la tradition assimilant la Sibylle ionienne à Daphné et au sanctuaire d'Apollon situé dans ce même lieu proche d'Antioche. Il aurait peut-être été utile de discuter ces diverses hypothèses et d'expliciter les raisons de la préférence pour Antioche. La démonstration aurait à mon avis gagné encore en force de persuasion. Petit détail enfin : le poème Sur le quatrième consulat d'Honorius, attribué par "télescopage" à Rutilius Namatianus probablement en raison de la mention du De reditu suo de

5. Cf. Bruno Bleckmann, «Ein Kaiser als Prediger : Zur Datierung der Konstantinischen "Rede an die Versammlung der Heiligen" ", Hermes, 125, (1997), p. 183-202 ; Michael Edwards, dans Michael Edwards, Martin Goodman, Simon Price (éds.), Apologetics in the Roman Empire. Pagans, Jews, and Christians, Oxford, 1999, p. 251-275 ; Harold Allen Drake, Constantine and the Bishops. The Politics of Intolerance, Baltimore, 2000 (Baltimore-Londres, 2002) ; Timothy D. Barnes, "Constantine's Speech to the Assembly of the Saints: Place and Date of Delivery", Journal of Theological Studies, 52 (2001), p. 26-36 ; Maria R. Cataudella, "Costantino, Giuliano e l'Oratio ad Sanctorum Coetum », Klio, 83 (2001), p. 167-181, pour des prises de position récentes. Dernièrement, Klaus Martin Girardet est revenu sur la question dans un ouvrage collectif sur Constantin et conclut que le Discours a été prononcé à Trèves le vendredi Saint 314 («Konstantin und das Christentum: Die Jahre der Entscheidung 310 bis 314 », dans A. Demandt, J. Engemann (éds.), Konstantin der Große. Geschichte - Archäologie - Rezeption, Trèves, 2006, p. 69-81, spécialement p. 76-80). 
cet auteur à la même note 83 , doit être restitué à Claudien, cité à la note suivante.

Une large place est faite ensuite aux relectures médiévales de la Sibylle. Comme on le sait, celle-ci a non seulement inspiré les poètes, mais aussi les compositeurs de musique, et cela dès le $\mathrm{IX}^{\mathrm{e}}$ siècle, où l'acrostiche sibyllin rapporté par Augustin dans la Cité de Dieu (XVIII, 23, 1) et repris dans un sermon longtemps attribué à l'évêque d'Hippone fait son entrée dans la liturgie de la nuit de Noël, avant d'être traduit en langues vernaculaires et d'être inséré dans des représentations théâtrales sacrées : l'ordo prophetarum. Partant d'un manuscrit du $\mathrm{X}^{\mathrm{e}}$ siècle provenant de Limoges (le BnF Lat. 1154) et contenant des neumes proto-aquitains, Marie-Noël Colette commente ce cantus Sibyllae dans une étude (Le chant de la Sibylle, composition, transmission et interprétation, p. 165-176), qui précise et complète les travaux, déjà anciens, de Solange Corbin, et ceux, plus récents, de Maricarmen Muntané Gómez. Particulièrement pertinente me paraît être l'association de ce chant de la Sibylle avec le thème de l'attente eschatologique, central dans l'acrostiche et si prégnant tout au long du haut Moyen Âge, et particulièrement aux approches de l'An Mil ${ }^{6}$. Suggestive aussi, l'explication selon laquelle les signes annonciateurs de la parousie, sombres et terrifiants, ne peuvent s'exprimer que dans une mélodie sobre et un chant minimal faisant toute la place au texte et à son intelligibilité, ce qui n'exclut pas totalement les ornements, qui se multiplieront du reste au cours des siècles (dans la Sibilla Mallorquina du XV $\mathrm{XV}^{\mathrm{e}}$ siècle ou la Valenciana du $\mathrm{XVI}^{\mathrm{e}}$ siècle, que l'on peut entendre désormais dans les enregistrements de Jordi Savall et de la Capella Reial de Catalunya, Alia Vox, 1999) ${ }^{7}$. C'est en lien avec l'article de M.-N. Colette et comme

6. Cf. Alfons Puigarnau Torrelló, « Muerte e Iconoclastia en la Cataluña medieval », dans Milenio : Miedo y religión, Universidad de La Laguna (Tenerife, Islas Canarias), 3-6 de Febrero del 2000, accessible sur le site internet : $<w w w . u l l . e s /$ congresos/conmirel/puigarna1.htlm>.

7. On complétera la bibliographie de cet article par les deux volumes de Maricarmen Muntané Gómez : El Cant de la Sibila, Madrid, 1996-97, et la thèse de doctorat non publiée de Niobe O'Connor, A Study of the Sibyl Chant and its Dramatic Performance in the Spanish Church (Ninth to Sixteenth Centuries), Université de St Andrews, 1984. Pour l'acrostiche sibyllin, je me 
son prolongement théâtral qu'on lira la contribution de Denise Huë (La Sibylle au théâtre, p. 177-195), qui aborde précisément l'ordo prophetarum $^{8}$. La datation basse proposée pour le sermon pseudoaugustinien Contra Iudaeos, Paganos et Arianos - VII ${ }^{\mathrm{e}}$ siècle - ne me semble pas pouvoir être suivie, dans la mesure où il figure dans un manuscrit de la seconde moitié du vI $\mathrm{e}^{\mathrm{e}}$ siècle, le Taurinensis G. V. 26, contenant également un fragment de l'Adversus quinque haereses, longtemps attribué à Augustin, lui aussi. L'auteur probable de ces deux textes est Quodvultdeus, ami et correspondant d'Augustin, qui sera élu évêque de Carthage entre 437 et 453 . Mais quoi qu'il en soit de la paternité et de la date de composition de ce sermon, sa dette à l'égard de la Cité de Dieu, XVIII, 23 d'Augustin est totale, et l'acrostiche, de même que les autres vers sibyllins repris de Lactance qui y sont cités, auraient de toute façon été connus des médiévaux, comme l'atteste l'extraordinaire diffusion de la somme augustinienne à travers tout le Moyen Âge. Du reste, la tradition manuscrite révèle que ces vers sibyllins ont également circulé à cette époque sous forme de centons ${ }^{9}$. D. Hüe évoque encore d'autres textes, écrits en français, comme le Girouflier aux dames, qui figure à la fin du Mistère du Vieil Testament, ou le Mistère de la Sibylle et d'Octovien (p. 185-190), et enfin le Mystère de l'Incarnation et Nativité de Nostre Seigneur et Redempteur Jésus-Christ, représenté

permets de renvoyer à mon étude sur «Augustin, les sibylles et les Oracles sibyllins », dans Augustinus afer. Saint Augustin : Africanité et universalité. Actes du colloque international, Alger-Annaba, 1-7 avril 2001, textes réunis par PierreYves Fux, Jean-Michel Roessli, Otto Wermelinger (Paradosis 45/1), Fribourg, 2003, p. 263-286.

8. Sur cette question, on complétera la bibliographie par les titres suivants : Noeli Kilmer, Ordo prophetarum: A Study and Transcription of the Medieval Liturgical Music-Drama (Thèse de doctorat défendue à la Catholic University of America en 1975) ; Dorothy F. Glass, "L'Ordo prophetarum en Italie », Cahiers de civilisation médiévale, 44 (2001), p. 259-273 (sur les représentations des prophètes dans l'Italie médiévale).

9. Cf. Bernhard Bischoff, « Die lateinischen Übersetzungen und Bearbeitungen aus den Oracula Sibyllina », dans Mélanges Joseph de Ghellinck, S. J., tome 1, Gembloux, 1951, p. 121-147 (= Mittelalterliche Studien. Ausgewählte Aufsätze zur Schriftkunde und Literaturgeschichte, t. 1, Stuttgart, 1966, p. 150171). 
à Rouen en 1474 (p. 190-192), dans lesquels la Sibylle devient « un personnage théâtral ». Isabelle His aborde pour sa part une étape ultérieure de la réception musicale de la Sibylle dans sa contribution sur La Sibylle en musique : d'Orlande de Lassus à Maurice Ohana (p. 255-267). L'attention de l'auteur porte avant tout sur le rapport subtil entre texte et musique, en partant des Prophetiae Sibyllarum de celui qu'on pourrait tout aussi bien nommer Roland de Lassus ${ }^{10}$ (sur le modèle de Roland Furieux de Tasse), comparées à deux œuvres d'un compositeur franco-espagnol né au Maroc en 1914 et mort à Paris en 1992, la Sibylle pour soprano, percussion et bandes (1968) et Trois prophéties de la Sibylle (1989), dont I. His se demande si elles n'ont pas été inspirées à ce dernier par le cycle des douze motets avec prologue de son prédécesseur à la Renaissance.

Les contributions suivantes s'intéressent à la place de la Sibylle dans la littérature française du Moyen Âge. En examinant les éléments parodiques des romans arthuriens du XIII ${ }^{\mathrm{e}}$ siècle - le Lancelot en prose (1220-1225), les Prophéties de Merlin (1276) et le Livre d'Artus (1280) -, Francine Mora explicite, dans son article intitulé La Sibylle séductrice dans les romans en prose du XIII siècle : une Sibylle parodique? (p. 197-209), la façon dont la Sibylle perd son caractère prophétique, virgilien et virginal (p. 206-207). Christine Ferlampin-Acher montre, dans sa Sebille prophétesse et maternelle : du monde antique au monde arthurien dans Perceforest (p. 211-225), comment, dans ce roman du XIV e siècle, la Sibylle devient, par des manipulations généalogiques complexes, ou la mère ancestrale du roi Arthur, ou la fée Morgane, ou la Dame du Lac. Fabienne Pomel (La Sibylle, guide et double de Christine dans l'autre monde des lettres "le Chemin de longue étude" de Christine de Pizan, p. 227239) examine cette brillante réécriture littéraire, inspirée de Jean de Mandeville, de Boèce et surtout de Dante. À l'instar de Virgile pour le poète florentin, la Sibylle de Christine de Pizan fonctionne de manière subtile - comme elle le fait du reste pour le poète latin

10. Plusieurs enregistrements sont disponibles, le dernier en date étant celui de l'Ensemble Daedalus, dirigé par Roberto Festa, 2006 (Alpha 095). On consultera aussi Philipp Weller, " "Notre Divin Orlande" : Lassus in His Time and in Ours », Early Music, 27/3 (1999), p. 493-497, 499. 
dans l'Énéide -, à la fois comme double de l'auteur, image de la sagesse, du savoir livresque, de la connaissance de l'écriture et de la poésie prophétique, et comme idéal du lecteur et emblème d'une voix transcendante. Vient ensuite une contribution sur le beau livre de Jean Rabel, Jean Dorat et Claude Binet: Sibyllarum duodecim oracula, publié en 1586. L'étude, précise et soignée, d'Emmanuel Buron, n'a malheureusement pas pu profiter d'un article de Salvatore Settis, « Sibilla Agrippa », paru, il est vrai, dans un périodique d'accès difficile : Études de lettres. Revue de la Faculté des Lettres. Université de Lausanne, 1985/4, p. 89-124. Cette contribution aurait permis à l'auteur de bénéficier de réflexions fort éclairantes sur l'iconographie des sibylles. Précisons enfin que le prénom du premier éditeur moderne des oracula Sibyllina (Bâle, 1545) est Xystus, et non Christus, Betuleius (forme latine de Sixt Birk ou Bircken, philologue augsbourgeois connu également comme auteur de pièces de théâtre inspirées par des thèmes bibliques à la manière des Mystères médiévaux).

Dans un article intitulé Sibylles fin de siècle (p. 273-283), Anne Ducrey fait résonner, pour clore le volume, quelques-uns des échos de la Sibylle dans le théâtre symboliste de Maurice Maeterlinck, Henri de Régnier, Alexandre Blok et Fernando Pessoa. On pourrait ajouter ici l'étrange et difficile sonnet de Gerard Manley Hopkins : Spelt from Sibyl's Leaves (1886), aux accents si profondément virgiliens ${ }^{11}$, et les poèmes que Marina Tsvetaïeva consacra à la Sibylle ${ }^{12}$ dans le sillage de son ami Rainer Maria Rilke ${ }^{13}$ au début $\mathrm{du} \mathrm{XX}^{\mathrm{e}}$ siècle. On pourrait également rappeler que l'un des poèmes les plus influents de la littérature anglo-américaine du $\mathrm{Xx}^{\mathrm{e}}$ siècle, The Waste Land de T. S. Eliot (1922) - traduit pour la première fois

11. De même que son modèle, le recueil intitulé Sibyl's Leaves (1817) de Samuel Taylor Coleridge.

12. Marina Tsvetaïeva, Стихотборения и поэмы ятитомах, 5 vols, New York, Russica, 1980-1993, ici vol. 3, p. 24-26 (traduction française dans M. Tsvetaïeva, Poésies, Paris, 1993, p. 43). Sur ce sujet, voir Olga Peters Hasty, Tsvetaeva's Orphic Journeys in the Worlds of the Word (Studies in Russian Literature and Theory), Evanston, 1996, spécialement p. 83-109.

13. Cf. «Eine Sibylle» (écrit entre le 22 août et le 5 septembre 1907 à Paris), dans Neue Gedichte II, 1908. Dans ce recueil, le poème « Eine Sibylle » est immédiatement précédé de « Ein Prophet» et de « Jeremia ». 
en français par Jean de Menasce en $1926^{14}$-, est placé sous le patronage de la Sibylle, dont il met en exergue les paroles prêtées à Trimalcion dans le Satiricon 48, 8 de Pétrone: "Et la Sibylle, donc ! À Cumes, je l'ai vue moi-même de mes yeux suspendue dans une bouteille, et quand les enfants lui demandaient: "Sibylle, que veux-tu?", elle répondait : "Je veux mourir." "

L'ouvrage, dont il faut souligner la confection soignée, s'achève par une très bonne bibliographie, distribuée en autant de parties qu'il y a de périodes couvertes par les contributions réunies. Mon seul regret est que ce livre, qui fait la part belle au monde romain, ne consacre pas plus de place aux oracula Sibyllina de composition juive et chrétienne. Même s'il n'avait pas pour ambition de livrer une synthèse sur le problème des sibylles, ce volume aurait gagné encore en intérêt s'il avait aussi tenu compte de cet aspect de la survie et de l'appropriation du langage oraculaire dans les mondes juif et chrétien. Peut-être cette lacune s'explique-t-elle par les limites imposées aux éditeurs. Pour s'en faire une idée, le lecteur se reportera aux introductions et traductions des parties juives et chrétiennes du corpus des Oracles sibyllins, parues, pour les premières, dans le volume d'André Dupont-Sommer et Marc Philonenko, La Bible. Écrits intertestamentaires (Bibliothèque de la Pléiade), Paris, 1987, p. 1035-1140 (traduction de Valentin Nikiprowetzky), et, pour les secondes, dans le tome II des Écrits apocryphes chrétiens, publié en septembre 2005 (deuxième tirage en août 2006) sous la direction de Pierre Geoltrain et Jean-Daniel Kaestli (Bibliothèque de la Pléiade), p. 1045-1083 (traduction de l'auteur des présentes notes critiques).

Le programme du colloque de Rennes annonçait en outre une communication de Patrizia Castelli intitulée « Triomphes des dieux sur les murs : la "construction" des images des dieux et des sibylles entre 1400 et $1500 »$, dont le texte ne figure pas dans les Actes. Cet exposé portait peut-être sur les sibylles du Tempio Malatestiano de Rimini ou sur celles, presque aussitôt détruites, du Palais du cardinal Orsini à Rome. De cette historienne de l'art, active à Ferrare, on lira

14. Cf. mon « Jean de Menasce et T. S. Eliot», dans Michel Dousse, JeanMichel Roessli (éds.), Jean de Menasce (1902-1973), Fribourg, 1998, p. 39-53 et 205-225. 
avec profit " "Solvet saeclum in favilla". Le immagini delle Sibille al servizio dell'ideologia », dans Maria Chiabò - F. Doglio (éds.), Mito e realtà del potere nel teatro : dall' antichità classica al Rinascimento. Convegno di studi, Roma 29 ottobre-1 novembre 1987 (Centro studi sul teatro medioevale e rinascimentale [Viterbo]. Convegno di studi 11), Rome, 1988, p. 313-332 et «Fonti ed immagini : le dieci sibille ovvero l'ideologia del potere politicoreligioso tra medioevo e rinascimento ", publié dans les Actes du colloque de Macerata, cités ci-dessus en note 4, p. 709-739. Enfin, deux intervenants ont dû se désister au dernier moment : Carla Di Francesco, qui devait parler des «Sibylles de la Casa Romei», dont elle a dirigé la restauration et qui avaient déjà fait l'objet de deux publications de sa part (« Le Sibille di Casa Romei : la proposta di una nuova interpretazione della funzione e del significato dei celebri affreschi quattrocenteschi », Ferrara : voci di una città $5 / 8$ [1998] 8-16 et Le Sibille di Casa Romei : storia e restauro, Ravenne, 1998), et Roberto Guerrini, qui avait annoncé une conférence sur «Les sources antiques du pavement du Dôme de Sienne » et dont les résultats avaient déjà été publiés sous le titre « Le Divinae Institutiones di Lattanzio nelle epigrafi delle Rinascimento. Il Collegio del Cambio di Perugia e il pavimento del Duomo di Siena (Ermete Trismegisto e Sibille) », Annuario dell'Istituto Storico Diocesiano di Siena 1 (1992-1993) 5-38 ${ }^{15}$, à lire en lien avec la contribution suivante d'Anna Maria Romaldo : «Corpus Titulorum Senensium. Le Divinae Institutiones di Lattanzio e il pavimento del Duomo di Siena » (p. 51-81) ${ }^{16}$. Entre-temps, le même R. Guerrini a fait paraître avec Marilena Caciorgna un magnifique ouvrage sur le pavement de la cathédrale de Sienne (Il pavimento del duomo di Siena. L'arte della tarsia marmorea dal XIV al XIX secolo : fonti e simbologia, Silvana, 2004), dans lequel un chapitre porte sur «Ermete e le sibille.

15. Repris sous une forme abrégée dans Senio Bruschelli (éd.), Il Duomo come libro aperto : leggere l'arte della Chiesa [Quaderni dell'Opera, Anno I numero 1], Sienne, 1997, p. 51-66.

16. Les deux études ont fait l'objet d'un compte rendu de Daniela Fausti dans le Bullettino Senese di Storia Patria, 104 (1997), p. 567-570. 
Il primo riquadro della navata centrale e le tarsie delle navate laterali » (p. 13-51).

Il est piquant de noter qu'une semaine plus tôt s'est tenue, non loin de Rennes, une autre rencontre scientifique sur les Sibylles, organisée par Alfrieda et Jackie Pigeaud dans le cadre des VIII Entretiens de La Garenne Lemot et dont les Actes ont été publiés à Nantes en août 2005.

Ayant moi-même participé à ces Entretiens, je ne suis pas le mieux placé pour en discuter le détail et me bornerai donc à une présentation générale et à quelques compléments bibliographiques. À la différence de la rencontre de Rennes, celle de Nantes ne visait pas à offrir un panorama exhaustif du traitement de la Sibylle à travers les âges. Il s'agissait plutôt de favoriser des échanges sur divers aspects de la question. Et, à cet égard, on peut dire que les organisateurs ont atteint leur objectif. Les communications présentées furent en effet très diverses, par leur contenu comme par leur forme et, malgré l'impression d'éclatement qui peut se dégager de l'ensemble et qui est en grande partie due à l'approche résolument interdisciplinaire de J. Pigeaud, les discussions qui entrecoupèrent les exposés furent au moins aussi nourries que ceux-ci.

Empêchés, pour des raisons familiales, d'animer ces Entretiens de leur présence, Alain Michel et Pierre Brunel ont eu la bonne idée de communiquer leur texte aux éditeurs, alors que d'autres, à l'instar de Pascal Griener et Brenno Boccadoro, ont préféré y renoncer, malgré d'intéressants exposés sur L'effacement de la Sibylle chez Ruskin pour le premier et les Prophetiae Sibyllarum de Roland de Lassus pour le second. Sous le titre «À propos des Sibylles : la connaissance de Dieu, l'extase et les extra-lucides » (p. 7-25), A. Michel nous livre une dissertation savante et richement documentée sur le thème de l'inspiration divine à travers les âges. Pierre Brunel, spécialiste bien connu de littérature comparée, brosse en fin de parcours un tableau complet de « La figure claudélienne de la Sibylle» (p. 191-201). Philippe Maréchaux (p. 27-36) nous fait bénéficier pour sa part d'une lecture rafraîchissante de la Sibylle de Panzoust de Sieur Rabelais, parodie ô combien drôle et subtile des commentaires humanistes sur les sibylles et leurs 
prophéties ${ }^{17}$. Dans ses « Notes sur la représentation des sibylles dans l'art italien » (p. 37-47), Édouard Pommier nous offre un intéressant tour d'horizon de l'iconographie des sibylles, tour d'horizon qui profiterait encore davantage aux lecteurs si les œuvres des Pisano, Ghiberti, Signorelli et autres Ghirlandaio, Pérugin et Raphaël qu'il commente avaient pu être reproduites. Le titre minimaliste de la contribution suivante ( $«$ Notule sur un tableau d'Antoine Caron », p. 49-53) ne doit pas être pris au premier degré, car son auteur, Yves Hersant, donne de cette œuvre, reproduite en fin d'article, une interprétation qui, si on peut la suivre, se révèle éminemment séduisante. Deux contributions sont ensuite consacrées à Corinne ou l'Italie de Madame de Staël, grande admiratrice de la Sibylle du Dominiquin, elle-même figurée sous les traits de la Sainte-Cécile de Raphaël, comme si, pour le Dominiquin, voix prophétique et inspiration musicale fondaient l'une dans l'autre et avaient la transcendance pour source commune. Michel Delon intitule sa contribution : «Corinne et la Sibylle, ou de l'engagement à la mélancolie » (p. 55-65), Blandine de Saint-Girons : «Du génie féminin : Corinne ou "la sibylle triomphante" » (p. 67-82 + 3 pls.). Bien que portant sur une même œuvre littéraire, les deux études ne font pas double emploi et jettent sur cette appropriation de la Sibylle à l'âge des Lumières un regard ma foi fort éclairant. Jean-Yves Boriaud s'arrête ensuite sur « Les Sibylles et la Renaissance romaine » (p. 83-91). L'auteur s'intéresse tout particulièrement à la période qui va de 1480 à 1520, qui voit apparaître le traité de Filippo Barbieri, Discordantiae nonnullae inter sanctos Hieronymum et Augustinum, dans lequel les sibylles, au nombre de douze et non plus de dix, « font l'objet d'une "description" systématique » (p. 86), qui va durablement influencer l'iconographie de nos prophétesses, même si on en a parfois exagéré la portée. Sur le ton lyrique qu'on lui connaît, Philippe Heuzé, en amoureux inconditionnel et transi du poète latin, cherche à montrer, dans sa «Sibylle selon Virgile » (p. 93-98), comment celui-ci a transformé, dans l'Énéide, la prêtresse

17. Pour un point de vue différent et complémentaire, cf. Florence M. Weinberg, "Written on the Leaves: Rabelais and the Sibylline Tradition", Renaissance Quarterly, 43/4 (1990), p. 709-730. 
du dieu Apollon en une initiée de l'au-delà, capable de guider le héros troyen dans sa catabase, tout comme Virgile servira plus tard de guide à Dante dans sa traversée des enfers ${ }^{18}$. Étienne Wolff aborde ensuite «Lactance et les Oracles sibyllins» (p. 99-106). Son étude, un peu rapide et superficielle, mériterait d'être reprise et approfondie, et surtout débarrassée des préjugés par trop simplistes de l'auteur vis-à-vis de la littérature paléochrétienne. Anna Maria Babbi s'interroge ensuite sur «La Sibylle dans les traductions françaises du "Guerrin Meschino" » d'Andrea da Barberino (p. 107121). Sous le titre «Révéler ce qui doit devenir l'évidence! Postures de la découverte mathématique à l'âge classique », Jean Dhombres, lui aussi absent de ces VIII ${ }^{\text {e }}$ Entretiens de La Garenne Lemot, nous livre (p. 123-150) une intéressante réflexion d'historien des sciences sur le débat autour de « la part d'inspiration que comporte la découverte scientifique » (p. 126). Je signale au passage que le cercle des savants anglais dans lequel évoluait Isaac Newton était si vivement intéressé par les Oracles sibyllins que certains d'entre eux se disaient prêts à les voir figurer dans le canon biblique. William Whiston par exemple, successeur de Newton à la chaire de mathématique de Cambridge de 1702 à 1710, a même été le premier, après John Floyer, à traduire les Oracles sibyllins en langue anglaise ${ }^{19}$.

Spécialiste de littérature espagnole du $\mathrm{XX}^{\mathrm{e}}$ siècle, Joceline AubéBourligueux s'est penchée sur les rencontres de Federico García Lorca avec la Sibylle-Sphinx : à la croisée des chemins de la culture

18. La bibliographie fournie dans cet article étant réduite à sa plus simple expression, le lecteur trouvera toutes les informations utiles dans Enrico Flores, «Sibilla », Enciclopedia virgiliana, t. IV, Rome, 1988, p. 825-827. J'ajouterai encore le titre suivant : Harold Mattingly, "Virgil's Golden Age: Sixth Aeneid and Fourth Eclogue", The Classical Review, 48 (1934), p. 161-165.

19. John Floyer, The Sibylline Oracles translated from the best Greek copies and compar'd with the sacred prophesies, especially with Daniel and the Revelations, and with so much history as plainly shews, that many of the Sibyl's predictions are exactly fulfill'd. With answers to objections made against them, Londres, 1713 ; William Whiston, A Vindication of the Sibylline Oracles, to which are added the Genuine Oracles themselves, with the Ancient Citations from them in their Originals and in English, and a few brief notes, Londres, 1715. 
antique et de la tradition andalouse (p. 151-172) ${ }^{20}$. Dans son « Portrait de l'artiste en Sibylle » (p. 173-179), Philippe Junod égrène quelques remarques intéressantes sur l'idéal de l'artiste dans la peinture de la Renaissance, inspirées par les modes de représentation des sibylles. L'article de Jackie Pigeaud (« La Sibylle de Pierre Petit », p. 179-190) aborde enfin un auteur et une œuvre largement méconnus des non modernistes : le De Sibylla libri tres de Pierre Petit (1617-1687), philosophe et médecin parisien, pour qui la Sibylle est une et ne saurait se démultiplier. On saura gré à l'initiateur des Entretiens de La Garenne Lemot d'avoir attiré l'attention du lecteur sur cet érudit du XVII siècle et d'avoir ainsi donné accès à quelques-unes de ses réflexions sur la prophétesse antique, la mélancolie et sa sœur pythienne. À ce propos, il ne sera pas sans intérêt de préciser que les sibylles et les oracles circulant sous leur nom ont occupé une place de choix dans les débats intellectuels de l'époque moderne (env. 1500-1800). Ceux-ci portaient avant tout sur des questions d'ordre théologique - statut des sibylles et des Oracles sibyllins par rapport aux prophètes de l'Ancien Testament et à la Révélation biblique, etc. -, mais ils débordaient aussi ce cadre pour toucher à des problèmes d'ordre littéraire, philosophique et médical, ce dont Pierre Petit fournit un excellent exemple. Les textes à explorer dans ce domaine sont innombrables et le travail, pour l'essentiel, reste à faire $^{21}$. Le volume s'achève enfin (p. 203-230 + pl.) sur une étude

20. Le titre exact est « À la croisée des chemins de la culture antique et de la tradition andalouse : les rencontres de Federico García Lorca avec la Sibylle-Sphinx ».

21. J'y ai consacré une partie de ma thèse de doctorat, qui paraîtra dans la Series apocryphorum du Corpus christianorum, sous le titre Les Oracles sibyllins (Livres I, 324-400 ; VI; VII et VIII, 217-500). Origines païennes et appropriations chrétiennes). Voir aussi Anthony Grafton, "Higher Criticism Ancient and Modern: The Lamentable Deaths of Hermes and the Sibyls", dans A. C. Dionisotti, Anthony Grafton, Jill Kraye (éds.), The Uses of Greek and Latin. Historical Essays (Warburg Institute Surveys and Texts 16), Londres, 1988, p. 155-170 (reproduit avec quelques changements sous le titre "The Strange Deaths of Hermes and the Sibyls", dans Anthony Grafton, Defenders of the Text. The Traditions of Scholarship in an Age of Science, 1450-1800, Cambridge (Mass.)-Londres, 1991, p. 162-177) ; Rieuwerd Buitenwerf, Book III of the Sibylline Oracles and its Social Setting. With an Introduction, Translation, 
détaillée du sixième livre des Oracles sibyllins, un hymne au Christ, appelé ici Fils de l'Immortel, qui pourrait bien constituer l'un des plus anciens poèmes chrétiens de langue grecque en dehors du Nouveau Testament (hymne aux Philippiens, par exemple). Étant l'auteur de cette contribution, je laisserai au lecteur le soin d'en juger.

En guise de conclusion, je crois pouvoir dire que malgré une certaine négligence dans la préparation du manuscrit et une mise en page qui s'apparente davantage à un mémoire de fin d'études qu'à un véritable livre, les études réunies par J. Pigeaud présentent dans l'ensemble un grand intérêt et ont de quoi sustenter les esprits. Elles compléteront en outre utilement le volume publié à Rennes un an plus tôt. Pour cela, il faut espérer qu'un effort de diffusion leur permette de sortir de la confidentialité.

jean-michel.roessli@unifr.ch

and Commentary (Studia in Veteris Testamenti Pseudepigrapha 17), LeydeBoston, 2003, p. 5-28; Ralph Häfner, Götter im Exil. Frühneuzeitliches Dichtungsverständnis im Spannungsfeld christlicher Apologetik und philologischer Kritik (ca. 1590-1736) (Frühe Neuzeit 80), Tübingen, 2003 (comptes rendus de Martin Muslow, Christlicher Humanismus im Zeitalter der philologischen Kritik. Zu Ralph Häfners magistralem Werk Götter im Exil, URL : http://iasl.uni-muenchen.de/rezension/liste/Muslow3484365803_1375.html; Dieter Martin, Sehepunkte, 6 (2006), URL : http://www.sehepunkte.de/2006/ 01/9429.html ; Guido Giglioni, « Voci della Sibilla e voci della natura: divinazione oracolare in Girolamo Cardano », Bruniana \& Campanelliana, 11/2 (2005), p. 365-387 ; Margherita Palumbo, « La fortuna degli Oracula Sibyllina nel seicento », ibid., p. 493-508. Dans le cadre du même colloque sur Le parole del futuro. Profezia e poesia nell'étà moderna (Naples, 8-9 octobre 2004), où ont été présentées les deux contributions précédentes, Michaela Valente avait brièvement évoqué « Gli Oracula sibyllina nel Rinascimento », qui sera publié ailleurs dans un avenir proche. 